\title{
Mycobacterial Spindle Cell Pseudotumor of the Appendix Vermiformis in a Patient with AIDS
}

Carlos Alberto Basílio-de-Oliveira, Walter A. Eyer-Silva,
Heliomar de Azevedo Valle, Ana Lúcia Rodrigues,
Ana Luisa Pinheiro Pimentel and Carlos Alberto Morais-de-Sá

\author{
Departments of Pathology and Clinical \\ Immunology, Gaffrée e Guinle University \\ Hospital, Rio de Janeiro, RJ,Brazil
}

\begin{abstract}
Mycobacterial pseudotumor (MP) is a rare pathologic presentation of both Mycobacterium tuberculosis and non-tuberculous mycobacterial disease, hitherto reported to occur only in immunosuppressed patients with or without human immunodeficiency virus infection. This lesion shares close pathologic resemblance to certain mesenchymal neoplasms, particularly Kaposi's sarcoma (KS), from which it must be properly differentiated due to distinct prognosis and therapy. We report a case of MP obliterating the lumen of the appendix vermiformis in a 34-year-old patient who died of complications of AIDS at our hospital in Rio de Janeiro. A total of 24 cases of MP (including our patient) have been described in the literature. MP has been found especially in lymph nodes, but extranodal lesions have been described in the skin, spleen, lung, bone marrow, brain and, in our patient, the appendix vermiformis. We offer a review of the other 23 published case reports of MP in both HIV-infected and uninfected patients and discuss the pathologic features that differentiate MP from KS.
\end{abstract}

Key Words: HIV infection, Kaposi's sarcoma, Mycobacterium, pseudotumor, spindle cell.

A rare presentation of mycobacterial disease in immunocompromised patients with or without human immunodeficiency virus (HIV) infection occurs in the form of a tumor-like proliferation of spindle cells known as mycobacterial pseudotumor(MP) [1-14]. This lesion shares close pathologic resemblance to mesenchymal neoplasms, particularly Kaposi's sarcoma (KS), from which it must be properly differentiated. Awareness of this unusual pathologic presentation of mycobacterial disease highlights the importance of performing acidfast stains of lesions of spindle cell proliferation, especially in immunocompromised patients. The lymph nodes are the main site of involvement but isolated cases

Received on 12 February 2001; revised 13 March 2001.

Address for correspondence: Dr. Carlos Alberto Basílio-de Oliveira. M.D. Department of Pathology, Hospital Universitário Gaffrée e Guinle, Universidade do Rio de Janeiro. Rua Mariz e Barros, 775, Zip Code: 20270-004 Rio de Janeiro / RJ, Brazil.

The Brazilian Journal of Infectious Diseases 2001;5(2):98-100 (C) 2001 by The Brazilian Journal of Infectious Diseases and Contexto Publishing. All rights reserved. $1413-8670$ of extranodal disease have also been reported. We report a case of MP of the appendix vermiformis in a patient with AIDS. To the best of our knowledge, this is the first report of MP from that site.

\section{Case Report}

A 34-year-old HIV-infected male patient died in August, 1986, of complications of AIDS. He experienced a chronic illness that lasted 2 years. He also had a diagnosis of schizophrenia since the age of 15. During the course of the HIV disease, he had recurrent diarrhea that proved to be very difficult to treat at a time when antiretroviral agents were not yet available. Severe weight loss, recurrent oral and esophageal candidiasis, lymphadenopathy, anemia and hypoalbuminemia were also part of the clinical picture. A diagnosis of histoplasmosis was made based on lymph node biopsy, but treatment with amphotericin B led to little general improvement. Laboratory studies were remarkable for a transfusion-dependent anemia (hematocrit range 15\% to 30\%), hypoalbuminemia (2.4 
$\mathrm{mg} / \mathrm{dL}$ ) and elevated activity of alkaline phosphatase (190 U/L). No intestinal pathogens could be recovered. The patient died of overwhelming sepsis. Necropsy demonstrated disseminated histoplasmosis (liver, prostate, adrenals, lungs, lymph nodes, thyroid, skin) and non-tuberculous mycobacterial disease (liver, lymph nodes, spleen, skin, large and small intestine). Examination of the appendix vermiformis demonstrated an obliterated lumen due to an unusual proliferation of spindle cells with eosinophilic to granular cytoplasm and spindle or oval nuclei with admixed inflammatory infiltrate. Ziehl-Neelsen stain showed numerous acidfast bacilli. Although the precise species of mycobacteria could not be determined by culture, the demonstration of abundant, elongated, acid-fast bacilli within the cytoplasm and outside the spindle cells was consistent with a diagnosis of non-tuberculous mycobacterial disease (Figure 1).

\section{Discussion}

Since its first description in 1985, a total of 24 cases of MP (including the present one) have been reported in the literature [1-14]. The first report was made based on histologic examination of nodular skin lesions from a 54-year-old immunosuppressed cardiac transplant recipient, and the lesion was described as a "sporotrichoid Mycobacterium avium intracellulare pseudotumor with microscopic features of the histoid variety of lepromatous leprosy". One of these 24 patients was a child who died of complications of AIDS [2]. Among the 23 adult patients, 1 was of the female sex [3]. Four had non-HIV-related immunodeficiency: 2 were transplant recipients [1,9]; 1 had Hodgkin's disease [6]; and 1 was on steroid therapy for the treatment of sarcoidosis [14].

The lymph nodes were the site of involvement of MP in 15 of the 24 reported cases [2-6,11-13]. MP was found in extranodal sites in 8 cases: in the skin in 3 cases $[1,3,7]$, the spleen in 2 cases $[8,10]$, the lung in 1 case [9], the bone marrow in 1 case [4], the brain in 1 case [14] and from the appendix vermiformis in our patient. Of note, among the 4 patients with non-HIVrelated immunodeficiency $[1,6,9,14], 3$ patients had extranodal MP (skin, lung and brain). Only 1 case of MP was associated with M. tuberculosis disease: the lesion was found in the lung of a 32-year-old male patient who had multiple complications of insulin-dependent diabetes mellitus and was submitted to both renal and pancreatic transplants [9]. Another patient had a skin lesion reported to be caused by $M$. gordonae as a complication of AIDS [7]. All other cases of MP were reported to be caused by M. avium intracellular, with or without culture confirmation.

MP lesions must be appropriately distinguished from lesions such as mesenchymal neoplasms, especially KS. This distinction is of utmost importance due to different prognosis and therapeutic approaches. The use of acid-fast stains is the simplest way to differentiate MP from KS, and other conditions. However, as both mycobacterial disease and KS are common disorders among immunosuppressed patients, it would not be surprising if they were simultaneously found at the same site. In fact, Logani, et al., [13] recently reported the coexistence of what they called "Kaposi sarcoma with mycobacteria" in lymph nodes of 3 HIV-infected patients. Those patients had mycobacterial disease but not MP. The presence of acid-fast bacilli and spindle cells in the same lymph node would have suggested the diagnosis of MP "if attention was not paid to the presence of more classical areas of KS". KS lesions can usually be distinguished by the longer, more compact fascicles with intercellular slitlike spaces as well as a higher frequency of mitotic figures. KS lesions may also be associated with extravasated red blood cells. In contrast, the spindle cells in MP have a loose storiform pattern with more eosinophilic to granular cytoplasm $[5,11,13]$. Additionally, MP and KS have distinct immunohistochemical profiles, as spindle cells from MP stain with markers of its monocytic-histiocytic origin $[3,5,12,13]$. Logani, et al., [13] suggested that stains for S-100 protein and $\mathrm{CD}_{68}$, a histiocyte-associated antigen, are excellent markers for spindle cells in MP and help differentiate them from KS cells in more difficult cases. KS cells, in contrast, frequently stain with markers of $\mathrm{CD}_{34}$ and $\mathrm{CD}_{31}$ cells. 
Figure 1. 1A) Pseudotumor occluding the lumen of the appendix vermiformis (H.E., 63x). 1B) Spindle cells with eosinophilic to granular cytoplasm and oval nuclei. An admixed inflammatory infiltrate is also seen (H.E., 160x). Inset: numerous acid-fast bacilli within and outside spindle cells as revealed by Ziehl-Neelsen stain (400x).

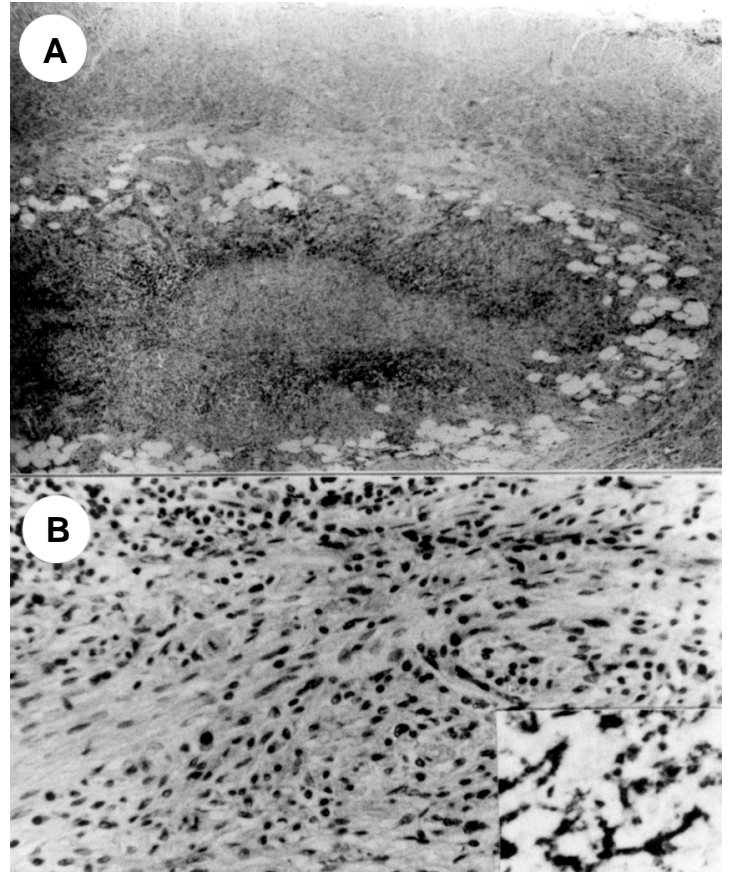

\section{References}

1. Wood C., Nickolov B.J., Todes-Taylor N.R. Pseudotumor resulting from atypical mycobacterial infection: a "histoid" variety of Mycobacterium avium-intracellulare complex infection. Am J Clin Pathol 1985;83:524-7.

2. Joshi V.V. Pathology of AIDS in children. Pathol Annu 1989;24:355-81.

3. Brandwein M., Choi H-S. H., Strauchen J., et al. Spindle cell reaction to nontuberculous mycobacteriosis in AIDS mimicking a spindle cell neoplasm - evidence for dual histiocytic and fibroblast-like characteristics of spindle cells. Virchows Archiv A Pathol Anat 1990;416:281-6.

4. Umlas J., Federman M., Crawford C., et al. Spindle cell pseudotumor due to Mycobacterium aviumintracellulare in patients with acquired immunodeficiency syndrome (AIDS). Positive staining of mycobacteria for cytoskeleton filaments. Am J Surg Pathol 1991;15:1181-7.

5. Chen K.T.K. Mycobacterial spindle cell pseudotumor of lymph nodes. Am J Surg Pathol 1992;16:276-81.

6. Apel R. Lymph node pseudotumor. Am J Surg Pathol 1993; $17: 91-2$.

7. Perrin C., Michiels J.F., Bernard E., et al. Cutaneous spindle cell pseudotumor due to Mycobacterium gordonae and Leishmania infantum: an immunophenotypic study. Am J Dermatopathol 1993; 15:553-8.
8. Suster S., Moran C.A., Blanco M. Mycobacterial spindle cell pseudotumor of the spleen. Am J Clin Pathol 1994; 101:539-42.

9. Sekosan M., Cleto M., Senseng C., et al. Spindle cell pseudotumors in the lungs due to Mycobacterium tuberculosis in a transplant patient. Am J Surg Pathol 1994; $18: 1065-8$.

10. Kumar S., Kumar D., Cowan D.F., Alperin J.B. Mycobacterial spindle cell pseudotumor of the spleen. Am J Clin Pathol 1994;102:863.

11. Corkill M., Stephens J., Bitter M. Fine needle aspiration of mycobacterial spindle cell pseudotumor. A case report. Acta Cytol 1995;39:125-8.

12. Wolf D.A., Wu C.D., Medeiros L.J. Mycobacterial pseudotumors of lymph node. A report of two cases diagnosed at the time of intraoperative consultation using touch imprint preparations. Arch Pathol Lab Med 1995; 119:811-4.

13. Logani S., Lucas D.R., Cheng J.D., et al. Spindle cell tumors associated with mycobacteria in lymph nodes of HIVpositive patients: "Kaposi sarcoma with mycobacteria" and "mycobacterial pseudotumor". Am J Surg Pathol 1999;23:656-61.

14. Morrison A., Gyure K.A., Stone J., et al. Mycobacterial spindle cell pseudotumor of the brain. A case report and review of literature. Am J Surg Pathol 1999;23:1294-9. 